\title{
On phase relation between toroidal and poloidal magnetic fields in the solar cycle 23
}

\author{
S. I. Zharkov ${ }^{1}$, Elena Gavryuseva ${ }^{2}$ and Valentyna V. Zharkova ${ }^{3}$ \\ ${ }^{1}$ Department of Applied Mathematics, University of Sheffield, Sheffield, UK \\ email: s.zharkov@sheffield.ac.uk \\ ${ }^{2}$ Arcetri Observatory/University of Florence, Florence, Italy, \\ email: elena@arcetri.astro.it \\ ${ }^{3}$ Department of Computing and Mathematics, University of Bradford, Bradford, UK, \\ email: v.v.zharkova@brad.ac.uk
}

\begin{abstract}
Phase relations is extracted at different latitudes between the weak background solar magnetic (poloidal) field and strong magnetic field associated with sunspots (toroidal field) by comparing low-resolution images from Wilcox Solar Observatory (WSO) and the high-resolution SOHO/MDI magnetograms. Sunspot areas and excess flux in all latitudinal zones (averaged with a sliding 1 year filter) reveal a strong positive correlation with the absolute and excess solar magnetic fields with a timelag of zero and $\sim 3$ years. The residuals of a sunspot magnetic excess flux averaged by one year from those by 4 years are shown to have well defined periodic temporal and spatial structures. The periods of these structures are close to $\pi / 4$ ( $\pi \approx 11$ years). The structures have maxima at $-40^{\circ}$ and $+40^{\circ}$ and reveal spatial drifts with time either towards the equator or the poles depending on a latitude of sunspot occurences.
\end{abstract}

Keywords. Sun: activity, Sun: sunspots, Sun: interior, Sun: magnetic field, methods: data analysis, methods: statistical.

\section{Introduction}

The solar cycle is believed to result from a hydro-magnetic dynamo that operates from the bottom of the solar convective zone (SCZ) in the solar interior where magnetic flux tubes are formed and travel for about a half of the cycle time of 11 years to the solar surface to appear as sunspots at the beginning of a new cycle (see reviews by Tobias, 2002; Ossendrijver, 2003; Krivodubskij, 2005). The mechanisms affecting a travel time of the magnetic tubes from the SCZ to the solar surface and their migration in latitudes and longitudes inside the solar interior define a duration of the solar cycle and the sunspot distribution on the solar surface. Sunspots are also shown to migrate in latitudes during the solar cycle with a minority of sunspots migrating towards the poles and the majority migrating towards the equator as shown in Maunder's butterfly diagrams (Maunder, 1904).

The groups with equatorward drifts have smaller tilt angles $\theta$ towards the equator while those moves towards the poles have higher tilts (Howard, 1991; Zharkova and Zharkov, 2007) . The groups with average tilt angles show a positive change of daily polarity separation $d$ between leading and following spots that also depends on a latitude $\phi$ as $\theta / d \approx-0.021 \phi-0.123$ (Holder et al., 2004; Zharkova and Zharkov, 2007).

The recent observations of the magnetic tilts of sunspot groups and active region areas and the total areas covered by sunspots and active regions in the Northern and Southern hemispheres are found to be periodic and very asymmetric during the cycle (Temmer et al., 2002; Zharkova and Zharkov, 2007). Distinct domination of one or another 
hemisphere is detected with the two basic periods: a long one of 11 years and a shorter one of about 2.5-3 years (or $\sim \pi / 4$ ) (Zharkov et al., 2005; Zharkov and Zharkova, 2006).

The opposite magnetic polarities of sunspot magnetic fields in the opposite hemispheres shown in the 'butterfly diagrams' in the cycles 20 and 21 allow to assume a full cycle $(\pi)$ lag during the cycle between the poloidal and toroidal fields (Stix, 1976; Vainstein et al., 1980). This can be achieved only if the angular velocity in decreasing with depth into the solar interior, i.e. $\frac{\partial \omega}{\partial r}>0$. However, a shape of the butterfly diagrams can be only reproduced by the dynamo models with an angular velocity increasing with depth (i.e. $\left.\frac{\partial \omega}{\partial r}<0\right)($ Stix, 1976$)$. This contradicts to the conclusion above deduced from a phase lag between toroidal and poloidal magnetic field, and this puzzle is not resolved yet.

On the other hand, some progress has been made with low-resolution observations of the solar magnetic field (SMF) from Wilcox Solar Observatory (WSO) showing the SMF itself to have a well defined four-zone structure: two polar zones with latitudes above $\pm 25^{\circ}$ and two pre-equatorial ones for latitudes ranging from zero to \pm 25 . The preequatorial zones have the opposite magnetic field polarities in the Northern and Southern hemispheres and they are swapped every 2-3 years (Gavryuseva, 2006) similar to those detected for sunspots and active regions (Zharkov et al., 2005; Zharkov and Zharkova, 2006).

Therefore, further investigation is required to establish the correlation between the appearances in latitude of magnetic flux tubes, or sunspots, and the variations of SMF in the opposite hemispheres during the different phases of the cycle.

\section{Data description}

For the purpose of the current study, we consider the averaged magnetic fields for sunspots and background solar surface within the $30 \mu$-hemispheres in the heliographic latitude sines (from 75.2 degrees North to 75.2 degrees South).

\subsection{Solar Feature Catalogue}

The searchable Solar Feature Catalogues (SFCs) with a 9 year coverage (1996-2005) are developed from digitized solar images using automated pattern recognition techniques (Zharkova et al., 2005a). The automated techniques were applied for detection of sunspots, active regions, filaments and line-of-sight magnetic neutral lines in full disk solar images in Ca II K1, Ca II K3 and Ha lines taken at the Paris-Meudon Observatory and white light images and magnetograms from SOHO/MDI. The catalogue contains 368 676 sunspots extracted from the $10082 \mathrm{SOHO} / \mathrm{MDI}$ images and around 100000 active regions starting from 19-05-1996 19:08:35 to 31-05-2005. The results of the automated recognition were verified with the manual synoptic maps and available statistical data revealing a high detection accuracy of $4-8 \%$ (Zharkov et al., 2005). Based on the recognized parameters, a relational database of Solar Feature Catalogues was built for every feature and published on the Bradford University web site http://solar.inf.brad.ac.uk/.

\subsection{Wilcox Solar Observatory (WSO) data}

We also use low resolution magnetograms captured daily with the WSO's Babcock solar magnetograph http://wso.stanford.edu/synoptic.html) measuring the line-of-sight component of the background solar magnetic field, thereafter, solar magnetic field (SMF). Each longitudinal value is the weighted average of all the observations made in the longitudinal zone within 55 degrees around the central meridian (Hoeksema, 1985). 


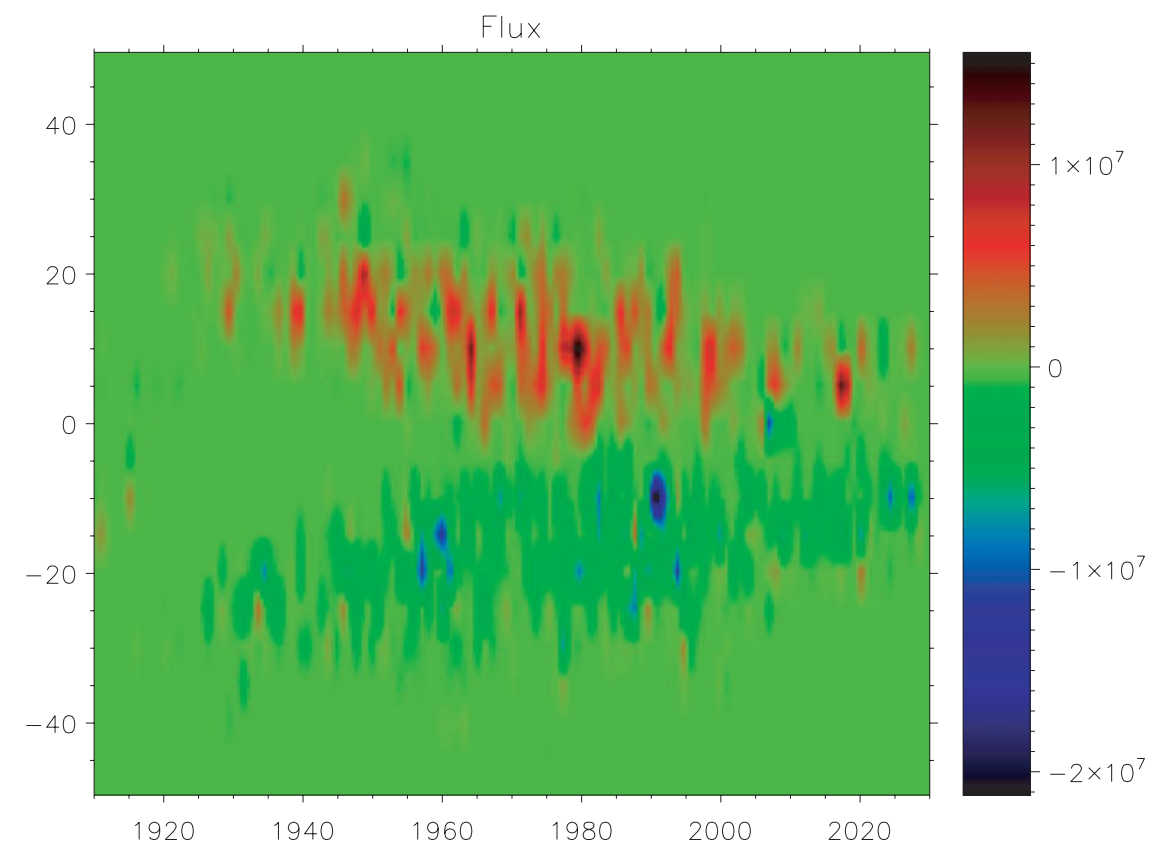

Figure 1. The butterfly diagrams in the cycle 23 for total sunspot daily areas (upper plot) and excess magnetic fields (lower plot) summarised for all the longitudes within narrow latitude strips of 2 degrees. The areas are measured in squared degrees and magnetic field in Gauss. CR number of 1910 corresponds to the start of 1996 and 2040 to the spring of 2005 .

\section{Results and Discussion}

\subsection{Quasi-3D butterfly diagrams}

Latitudinal variations of sunspot excess magnetic flux (EMF) calculated in the narrow latitude strips for the whole longitude range in different Carrington rotations are presented in Figure 1. These are similar to the classic Maunder's butterfly diagrams for sunspot appearances (Maunder, 1904; Hathaway, 2005) but enhanced by a third dimension associated with the excess magnetic flux from the areas covered by sunspots at different latitudinal strips and summated for all longitudes ( $\mu$-spheres). The total SMFs of sunspots at a given Carrington rotation are marked by a dark blue colour for the negative flux and by a green one for the positive flux.

The butterfly diagram for flux reveals a fine structure in latitude and time that appears during the activity period as vertical threads, or strips. These strips are larger in one hemisphere than the symmetric strip in the other hemisphere in one time interval and then in another one, the strip with larger area shifts to other latitude or even to the opposite hemisphere.

\subsection{Correlation of sunspot EMF and background $S M F$}

Since SMF is assumed to carry the poloidal magnetic field while sunspots or active regions are associated with the toroidal one, then, similarly to the analysis carried out by Stix (1976) a comparison of these time series will allow to establish the relations between these fields for different phases of the solar cycle 23 .

\subsubsection{A long-term time lag}

A comparison of these SMF variations with those for the sunspot EMF averaged by a one Carrington rotation (Figure 2) shows that during the whole cycle the both SMF and 


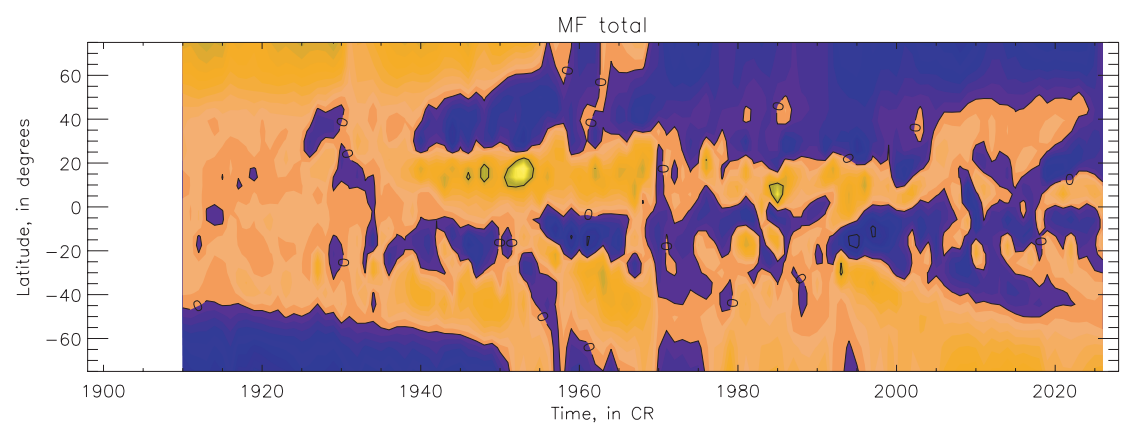

MF flux Sun Spots

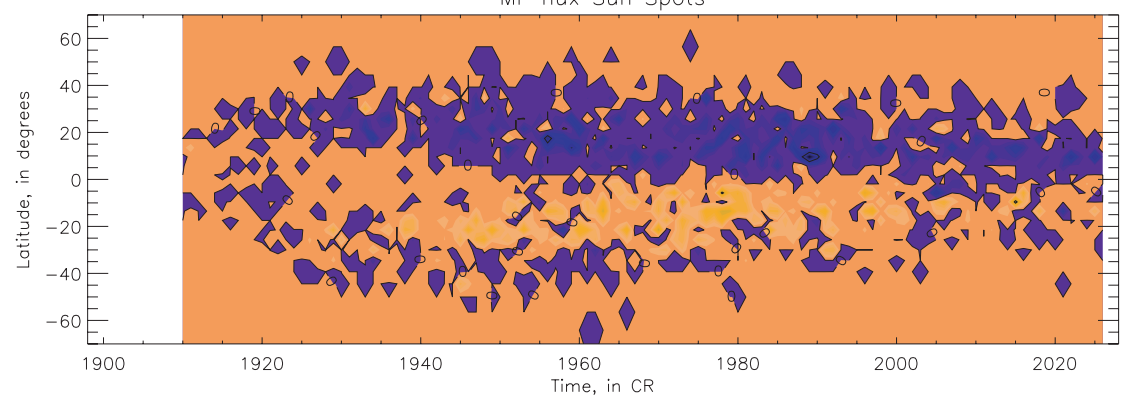

Figure 2. Latitudinal distributions of the excess SMF (upper plot) and sunspot excess fluxes (bottom plot) in the cycle 23 averaged by a 1 Carrington rotation filter. Blue-navy colour denotes the positive polarity and orange-yellow the negative one.

EMF fluxes have the opposite polarities in the same hemispheres while changing their signs in the other hemisphere. This confirms that a long-term phase relation between the weak poloidal (SMF) and strong toroidal (sunspots) magnetic field is close to the whole cycle period ( $\pi=11$ years), similar to the cycles 20 and 21 measured by Stix (1976).

In order for account for meridional flows of dynamo waves, i.e. to satisfy the condition $\alpha \frac{\partial \omega}{\partial r}<0$, this phase relation implies the helicity parameter $\alpha$ has to be positive (Krivodubskij, 2005). However, this contradicts to more recent studies where $\alpha$ is found changed its sign from positive near the surface to the negative at the bottom of SCZ, in order to allow the flux tubes to travel upwards (Yoshimura, 1981). This puzzle motivated us to look further for short-term relation between these magnetic fields.

\subsubsection{A short-term time lag}

The nature of short-term oscillations in the sunspot EMFs can be deduced from the quasi-3D butterfly diagrams averaged with the 1 one year (1y) and 4 years (4y) filters. The residuals of the sunspot fluxes (Figure 3) vary quasi-periodically during the cycle revealing either much lower (blue/navy colours) or much higher (green/brown colours) sunspot EMFs than those averaged for 4 year. Each pattern for every latitide band lasts for 2.5-3 years and their signs are changed to the opposite during the next 2.5-3 years period. These resemble the periodicities reported in the backgrouns SMF (Gavryuseva, 2006; Zharkov et al., 2008) that points out onto a link between EMF and SMF.

The period of these variations, $\sim \pi / 4$, are checked with a auto-correlation of the latitudinal distributions of sunspot EMF in the Northern and Southern hemispheres 

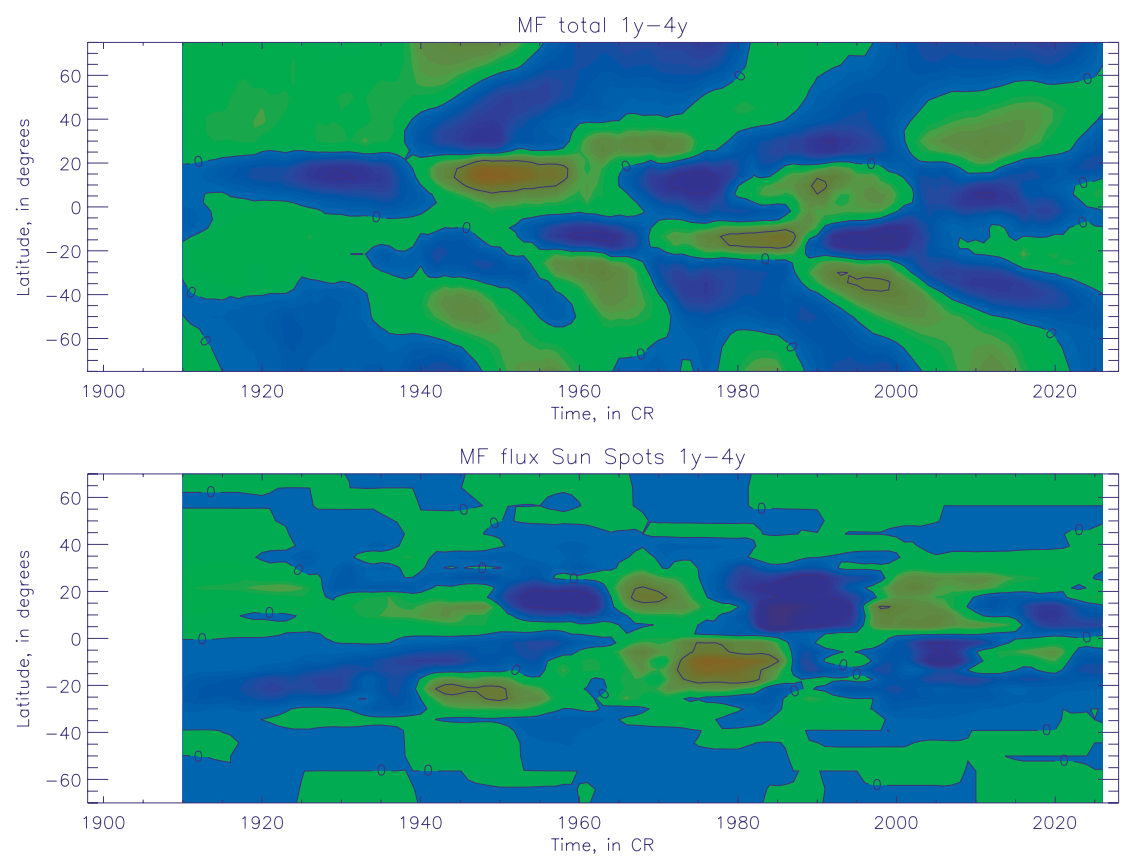

Figure 3. The residuals in the latitudinal distributions in the cycle 23 averaged by 1 year minus those by 4 years for excess SMF (upper plot) and excess sunspot flux (bottom plot). The green-brown colour presents the positive residuals and the blue-navy one denotes the negative ones (see Figure 1 and $\S 2$ for the relation between CR and years), the residuals are twice higher for inner contours that for outer ones.

averaged by 1 year and the results are presented in Figure 4. In order to establish links between EMF and SMF, the sunspots EMF averaged were correlated with the similar background SMF (see Figure 8 in Zharkov et al., 2008). The 1y-averaged areas show a strong positive correlation with the background SMFs around a zero timelag and a strong negative one at about 50 Carrington rotations (about 3 years, or $\pi / 4$ ).

The additional feature appears in the sunspot EMF is that the polarity of each periodic structure is opposite in every zone in each hemisphere and changes their signs in 2.5-3 years like changing colours in a chess board. The time lag increases from the polar zone in the Southern hemisphere through the equator to the polar zone in the Northern one.

The autocorrelation of EMF and the correlation of the residuals for the $1 \mathrm{y}-4 \mathrm{y}$ sunspot EMF and with those of SMF reveal the 4-zone latitudinal structure similar to those reported for the SMF (Gavryuseva, 2006): two pre-equatorial zones and two pre-polar zones measured from the top of the royal zones towards the equator or the poles, respectively. The variations of correlation ocefficients in the pre-equatorial zones correspond to the sunspot migration towards the equators seen as 'strips of the EMF butterfly diagrams (see $\S 3.1$ ), the similar variations in the pre-polar zones correspond to the sunpot migration towards the poles. This correlation changes its sign every 2.5-3 years similarly to the symmetric variations of the background SMF (Zharkov et al., 2008).

Basically, in the Carrington rotations 1930-1940 (years 1996-97) the polarity is negative in the Northern hemisphere and positive in the Southern one that after 2.5-3 years 


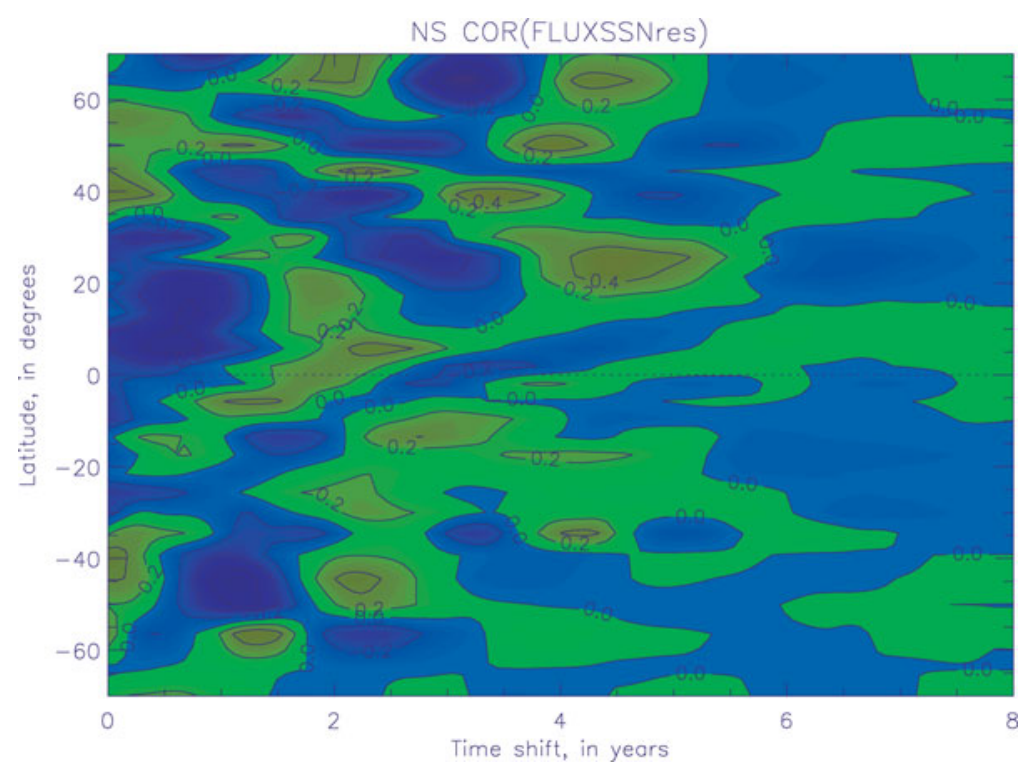

Figure 4. The C-correlation maps for the time lags in years between the $1 \mathrm{y}-4 \mathrm{y}$ residuals of the SMF and sunspot EMF in the Northern and Southern hemispheres. Numbers at the contours show the C-correlation coefficients.

changes to the opposite ones in each zone and the pattern reats every 2.5-3 years. Obviously, in some of these periods the polarity of SMF will be opposite to the leading polarity of sunspots while in others it will coincide with it.

This correlation lends support to a suggestion that there is also a shorter phase of about $\pi / 4=2.75$ years when the signs of the SMF polarity in each hemisphere are changed. These changes are likely to modulate the borders of the 'royal zone' in the solar interior where the magnetic pumping allows to flux tubes to float to the solar surface (see Figure 7 in Zharkov et al., 2008).

This, in turn, leads to the asymmetric fluctuations of the symmetric component of the poloidal field in the Northern and the Southern hemispheres (see Figure 6 in Stix, 1976). If the sign of the SMF in this period coincides with the leading polarity sign, the SMF suppresses a flux tube buoyancy and a frequency of the sunspot appearances, and vice versa, if this sign is opposite to the leading polarity, it increases the sunspot appearances and when it coincides with the leading polarity, it suppresses them.

\section{Conclusions}

In the present paper, a correlation was established between the appearances of sunspots at different latitude of each hemisphere and latitudinal variations of the solar magnetic field at different phases of the solar cycle 23.

The quasi-3D butterfly diagrams for the sunspot EMFs reveal a strip-like fine structure in latitudes appearing during the activity period. A period of about $2.5-3.0$ (or $\pi / 4$ ) year can be extracted from the strip variations of sunspot excess magnetic flux averaged over 1 year. This period is also confirmed by a strong positive correlation with the symmetric part of the background solar magnetic field (SMF) which reveals a well defined four zonal latitudinal structure in each hemisphere with the boundaries located along the tops of the 'royal zone'. 
During the whole cycle the overall magnetic polarities of EMF and SMF in the opposite hemispheres averaged over 1 Carrington rotation are opposite similar to those found for the cycles 20 and 21 (Stix, 1976). Although their magnetic polarities in the symmetric latitudinal zones of the Northern and Southern hemispheres averaged by 1 year are opposite and the signs are changed periodically every 2.5-3 years either coinciding with the leading polarity in a given hemispere or being opposite to it. This, in turn, leads to a period of $2.5-3$ (or close to $\pi / 4, \pi=11$ ) years when in one hemisphere its SMF favours the sunspot formation resulting in higher sunspot areas and EMF averaged by 1 year than in the other hemisphere; then the polarities swap and the sunspot areas and EMF in the other hemisphere become higher.

Therefore, the magnetic field confined in the sunspot EMF and background SMF reveals the two periods of their phase relation: a long term one of 11 years, or $\pi$ similar to those reported by Stix (1976) and a short-term period of about 2.5 -3 years $(\sim \pi / 4)$.

\section{References}

Gavryuseva, E. , 2006, News of the Academy of Sciences, Izv. RAN, Ser. Physics, 70, No.1, 102 Hathaway, D.H.: 2005, ASP-CS, 346, 19

Hoeksema, J.T., 1985, http://sun.stanford.edu

Holder, Z., Canfield, R.C. McMullen, R.A., Nandy, D., Howard, A.F. \& Pevtsov, A.A.:2004, ApJ, 611, 1149

Howard, R.F.: 1991, Solar Phys., 136, 251

Kitchatinov, L.L. and Rudiger,G : 1999, A\&A, 344, 911.

Krivodubskij, V.N.: 2005, Astron. Nachr., AN, 326, 61-74

Maunder, E.W. MNRAS, 64, 747, 1904

Nandi, N. \& Choudhuri, A.R.: ApJ, 551: 576, 2001

Ossendrijver, M.: The solar dynamo, A\&AR, 11 287, 2003

Stix, M.:1976, Differential rotation and the solar dynamo, MNRAS, 47: 243

Temmer, M., Veronig, A., Hanslmeier, A.: A $\& A$, 390: 707-715, 2002

Tobias, S.M.: 2002, Phil.Trans.R.Soc.London, 360, 2741

Vainstein, S.I., Zeldovich, Ya.B, Ruzmaikin, A.A,: 1980, Turbulent Dynamo in Astrophysics, Nauka, Moscow

Yoshimura, H..: 1981 ApJ, 247: 1102.

Zharkov, S.I. and Zharkova, V.V.: 2006, Adv. Sp. Res., 38 N5, 868

Zharkov, S. I., Gavryuseva, E. and Zharkova, V. V.: 2007, Solar Phys., in press

Zharkov, S. I., Zharkova, V. V. and Ipson, S. S.: 2005, Solar Phys., 228/1, 401

Zharkova, V. V., Ipson, S. S., Zharkov, S. I. Benkhalil, A., Aboudarham, J. and Fuller, N.: 2005a, Solar Phys., 228/1, 134

Zharkova, V. V., Zharkov, S. I. and Benkhalil, A.K.: 2005b, MemSAI, 75, 1072

Zharkova, V.V. and Zharkov, S.I.: 2007, Adv. Sp. Res., doi:10.1016/j.asr.2007.02.082 